\title{
Management of postnatal depression in primary care: a window of opportunity
}

The postnatal period is a particularly distressing time to experience mental health problems. Detection and appropriate treatment of psychiatric symptoms at this critical time in family life is perhaps more important than at any other time. Postnatal depression (PND) affects more than one in 10 women; ${ }^{1,2}$ it significantly impacts on partners; ${ }^{3}$ is associated with an impaired mother-infant bond; ${ }^{4}$ and has been linked to poor cognitive and emotional development in the infant, ${ }^{5}$ thus contributing to the inter-generational transmission of poor health.

Suicide is a leading cause of maternal death in the UK; the largest proportion due to PND and puerperal psychosis, ${ }^{6}$ and completed suicides are thought to be the tip of the iceberg in terms of 'near miss' events. However, many women are reluctant to disclose PND symptoms to healthcare professionals. ${ }^{7}$ Strategies for identifying PND and supporting women and their families are therefore very important and must be timely, holistic, and appropriate to new mothers.

\section{INTERVENTIONS AND GUIDELINES}

The perinatal period provides many opportunistic occasions within which to identify and manage mild, moderate, and severe mental illness. This is due to the increased level of health professional contact through routine antenatal and postnatal contact, which does not occur in the non-postpartum population. Although GPs are often the first port of call for women ${ }^{8}$ or their families seeking help during pregnancy and postnatally, all healthcare professionals in contact with pregnant or postpartum women should be alert to possible symptoms, either those of new onset or of a more chronic nature.

Given the evidence for the effectiveness of both pharmacological and psychological interventions in improving health outcomes when PND is identified, there is much to be gained from early identification and management in primary care. Guidelines from the National Institute for Health and Clinical Excellence (NICE) ${ }^{9}$ recommend that mild to moderate symptoms should be managed within primary care, with psychological therapies as a first-line treatment. NICE guidelines ${ }^{9}$ also recommend a lower severity threshold for treatment in the perinatal period due to the evidence for adverse, long-term effects on infant and child wellbeing. However, in the current economic climate, the most efficient method of providing access to such psychological support is unclear.

\section{SKILLS AND TRAINING}

In this issue of the BJGP, Slade and colleagues $^{10}$ report on a qualitative study embedded within the PoNDER trial, ${ }^{11}$ which investigated the benefits of specific training for health visitors in providing psychological interventions for PND. This study involved 4084 women, making it the largest published intervention trial for PND in a primary care setting.

In the Slade et al study, a sub-sample of 30 women with a high probability of PND, defined according to a cut-off point of $\geq 18$ on the widely used Edinburgh Postnatal Depression Scale, ${ }^{12}$ took part in a qualitative interview about their experiences of symptom identification and of being offered and accepting psychological interventions by health visitors.

The study found that women who accepted help reported positive experiences of psychological sessions, and experienced them to be beneficial and 'ideal care'. However, women who declined the sessions felt that they were unable to relate to their health visitor or did not know them well enough. The interpersonal skills of healthcare professionals and their ability to engage with mothers who may have mental health problems, either during pregnancy or after childbirth, may be the key to successful outcomes.

The study by Slade et al may provide useful insight for the training of health visitors or other healthcare professionals (for example, midwives) in detecting and managing PND. However, there are training implications that come at a time of economic restraint, which will be competing against other healthcare priorities. The findings from the study by Slade and colleagues also raise several related issues because the ideal time, location, or health professional group best placed to identify and manage the condition is unclear.

\section{CONTINUITY OF CARE}

The recently published King's Fund research paper on the role of GPs in maternity care ${ }^{13}$ properly asks the question of whether the trend to erode the role of GPs in maternity services will impact on care quality for perinatal mental health care. Although currently $83 \%$ of women still visit the GP when pregnancy is identified, ${ }^{8}$ increasingly they are encouraged to bypass the GP to book directly with a midwife. This approach poses some challenges.

The GP is the one professional with access to a complete and up-to-date history for each woman, and the communication of this relevant history could save lives. The Confidential Enquiry into Maternal Deaths ${ }^{6}$ found that in cases resulting in suicide, past psychiatric history was often not communicated between healthcare professionals. Furthermore, women may be uncomfortable disclosing a history of severe mental health problems, and midwives do not receive training to identify rare or complex mental health problems. GPs are well placed to raise awareness of PND with pregnant women, monitor mental health status during pregnancy, detect early symptoms of PND, and make urgent referrals to specialist secondary care services if severe mental 
illness is suspected.

There is a problem with continuity of care within existing UK maternity services. Compared to other health professionals, GPs tend to have greater continuity of care, which may result in a greater level of trust and prior knowledge, and hence may be important in identifying perinatal psychiatric symptoms.

Maternity services in the UK are organised in such a way that midwives whom women have known throughout their pregnancy usually hand over care to health visitors in the early postpartum days. A new trust must therefore be developed between health visitors and pregnant women at a time when symptoms of low mood may make the development of a trusting relationship difficult.

As Slade et al report, this relationship is all important: the interpersonal skills of healthcare professionals and their relationship with women experiencing mental health problems were key determinants of women's decision to accept help and to successful outcomes. Seeking help for mental health issues in the postpartum period is difficult. Women fear judgment, fear being regarded as unsuitable parents, are unsure about who is the appropriate health professional to seek help from, and are concerned about the level of expertise of healthcare professionals in discussing PND. ${ }^{7,10,14}$

\section{THE ROLE OF GPS IN TREATMENT}

The primary goals of improving perinatal mental health care must be to reduce the impact of psychiatric symptoms on the next generation; reduce the distress caused to women and their families; and to address maternal suicide rates. Reducing maternal suicide rates requires all healthcare professionals in contact with women in the postnatal period to be alert to suicidal ideation, hopelessness, and the symptoms of severe mental illness.

The GP role is much clearer when severe mental illness, such as severe depression, mania or psychosis, arises as GPs tend to be the primary gatekeepers for new referrals to secondary mental health services. Early symptoms of manic or psychotic illness can develop acutely in the postpartum days, with rapid escalation, and must be regarded as a true psychiatric emergency. Delayed treatment can result in risk to the safety of mother and infant and longer, more severe, and difficult-to-treat episodes.

The speed and appropriateness of action in such severe cases can impact greatly on patient experience. Severe, complex, or treatment-resistant mental illnesses should be referred to secondary services. Ideally, referral to a specialist perinatal psychiatrist or mother and baby unit is recommended,,$^{15}$ although not all regions have yet invested in such units.

While the responsibilities of the GP for general maternity care have been eroded, as highlighted by The King's Fund report, ${ }^{13}$ their responsibilities for perinatal mental health care are actually increasing. ${ }^{9}$ Training in the specialist issues surrounding pharmacological treatments in the perinatal period is needed; and unless funding is made available for improved access to psychological therapies, as proposed by $\mathrm{NICE}^{9}$ - whether delivered by GPs, specially trained health visitors, Improving Access to Psychological Therapies programme workers, or psychotherapists primary care may be unable to meet this increased responsibility for perinatal mental health care.

Until services for women in the perinatal period are designed to be approachable and flexible enough to overcome the barriers to disclosure of symptoms, our healthcare services will fail to meet the needs of women and families. Any proposed approaches to improving perinatal care must ensure that all healthcare professionals are equipped with appropriate skills and competencies, not only to detect and manage perinatal depression but also to encourage engagement and acceptance of treatment. In this way, childbearing women will be the beneficiaries of competent and empathetic health professionals who can elicit their concerns and engage them in treatment within a supportive, non-judgmental relationship.

\section{Lynda Tait,}

PhD, CSci, Research Fellow, University of Birmingham.

\section{Jessica Heron,}

PhD, Research Fellow, Perinatal Research Programme, Birmingham \& Solihull Mental Health NHS Foundation Trust, Birmingham.

\section{Provenance}

Commissioned; not peer reviewed.

\section{REFERENCES}

1. Evans J, Heron J, Francomb H, et al. Cohort study of depressed mood during pregnancy and after childbirth. BMJ 2001; 323(7307): 257-260.

2. O'Hara MW, Neunaber DJ, Zekoski EM. Prospective study of postpartum depression: prevalence, course, and predictive factors. J Abnorm Psychol 1984; 93(2): 158-171.

3. Lovestone S, Kumar R. Postnatal psychiatric illness: the impact on partners. Br J Psychiatry 1993; 163: 210-216.

4. Tronick E, Reck C. Infants of depressed mothers. Harv Rev Psychiatry 2009; 17(2): 147-156.

5. Murray L, Sinclair D, Cooper P, et al. The socioemotional development of 5-year-old children of postnatally depressed mothers. J Child Psychol Psychiatry 1999; 40(8): 1259-1271.

6. Lewis G. Why mothers die 2000-2002: the sixth report of confidential enquiries into maternal deaths in the United Kingdom. London: CEMACH; 2004.

7. Chew-Graham CA, Sharp D, Chamberlain E, et al. Disclosure of symptoms of postnatal depression, the perspectives of health professionals and women: a qualitative study. BMC Fam Pract 2009; 10: 7.

8. Redshaw M, Rowe R, Hockley C, Brocklehurst P. Recorded delivery: a national survey of women's experience of maternity care 2006. Oxford: University of Oxford, National Perinatal Epidemiology Unit, 2007. https://www.npeu.ox.ac.uk/recorded-delivery (accessed 6 Oct 2010).

9. National Institute for Health and Clinical Excellence. Antenatal and postnatal mental health. Clinical management and service guidance. London: National Institute for Health and Clinical Excellence; 2007.

10. Slade P, Morrell CJ, Rigby A, et al. Postnatal women's experiences of management of depressive symptoms: a qualitative study. Br J Gen Pract 2010; DOI:

10.3399/bjgp10X532611 (abridged text, in print: Br J Gen Pract 2010; 60: 829-836).

11. Morrell CJ, Slade P, Warner R, et al. Clinical effectiveness of health visitor training in psychologically informed approaches for depression in postnatal women: pragmatic cluster randomised trial in primary care. $B M J$ 2009; 338: a3045.

12. Cox JL, Holden JM, Sagovsky R. Detection of postnatal depression. Development of the 10-item Edinburgh Postnatal Depression Scale. Br J Psychiatry 1987; 150: 782-786.

13. Smith A, Shakespeare J, Dixon A. The role of GPs in maternity care - what does the future hold? London: The King's Fund, 2010.

14. Stanley N, Borthwick R, Macleod A. Antenatal depression: mothers' awareness and professional responses. Prim Health Care Res Dev 2006; 7: 257-268.

15. Royal College of Psychiatrists. Perinatal maternal mental health services (council report CR88). London: Royal College of Psychiatrists, 2000.

DOI: 10.3399/bjgp10X538930

\section{ADDRESS FOR CORRESPONDENCE}

\section{Lynda Tait}

University of Birmingham, Primary Care Clinical Sciences, School of Health \& Population Sciences, Primary Care Clinical Sciences Building, Edgbaston Birmingham, B15 2TT.

E-mail: I.tait.1@bham.ac.uk 